\title{
A STAT3-inhibitory hairpin decoy oligodeoxynucleotide discriminates between STAT1 and STAT3 and induces death in a human colon carcinoma cell line
}

Inès Souissi ${ }^{1,2}$, Patrick Ladam ${ }^{2,6}$, Jean AH Cognet ${ }^{7}$, Stéphanie Le Coquil ${ }^{1,2}$, Nadine Varin-Blank ${ }^{1,2}$, Fanny Baran-Marszak ${ }^{1,2,4}$, Valeri Metelev ${ }^{5}$ and Remi Fagard ${ }^{1,2,3^{*}}$

\begin{abstract}
Background: The Signal Transducer and Activator of Transcription 3 (STAT3) is activated in tumor cells, and STAT3inhibitors are able to induce the death of those cells. Decoy oligodeoxynucleotides (dODNs), which bind to the DNA Binding Domain (DBD) of STAT3, are efficient inhibitors. However, they also inhibit STAT1, whose activity is essential not only to resistance to pathogens, but also to cell growth inhibition and programmed cell death processes. The aim of this study was to design STAT3-specific dODNs which do not affect STAT1-mediated processes.

Results: New dODNs with a hairpin (hpdODNs) were designed. Modifications were introduced, based on the comparison of STAT3- and STAT1-DBD interactions with DNA using 3D structural analyses. The designed hpdODNs were tested for their ability to inhibit STAT3 but not STAT1 by determining: i) cell death in the active STAT3dependent SW480 colon carcinoma cell line, ii) absence of inhibition of interferon (IFN) $\gamma$-dependent cell death, iii) expression of STAT1 targets, and iv) nuclear location of STAT3 and STAT1. One hpdODN was found to efficiently induce the death of SW480 cells without interfering with IFN $\gamma$-activated STAT1. This hpdODN was found in a complex with STAT3 but not with STAT1 using an original in-cell pull-down assay; this hpdODN also did not inhibit IFN $\gamma$-induced STAT1 phosphorylation, nor did it inhibit the expression of the STAT1-target IRF1. Furthermore, it prevented the nuclear transfer of STAT3 but not that of IFN $\gamma$-activated STAT1.

Conclusions: Comparative analyses at the atomic level revealed slight differences in STAT3 and STAT1 DBDs' interaction with their DNA target. These were sufficient to design a new discriminating hpdODN that inhibits STAT3 and not STAT1, thereby inducing tumor cell death without interfering with STAT1-dependent processes. Preferential interaction with STAT3 depends on oligodeoxynucleotide sequence modifications but might also result from DNA shape changes, known to modulate protein/DNA interactions. The finding of a STAT3-specific hpdODN establishes the first rational basis for designing STAT3 DBD-specific inhibitors.
\end{abstract}

Keywords: Hairpin decoy oligodeoxynucleotide (hpdODN), STAT3, STAT1, Colon carcinoma cell line

\section{Background}

STAT3 belongs to the signal transducers and activators of transcription (STATs) family of transcription factors (TFs) [1]. STAT3 is activated in response to several cytokines and growth factors, including IL-6, IL-10, the

\footnotetext{
* Correspondence: remi.fagard@avc.aphp.fr

'INSERM, Unité 978, Bobigny 93017, France

Full list of author information is available at the end of the article
}

epidermal growth factor (EGF), and interferon (IFN) $\alpha$ and is also weakly activated in response to other cytokines, including IFN $\gamma$ in some cellular contexts [2]. Activation of STAT3 involves phosphorylation of tyrosine 705 by cytokine receptor-associated Janus Kinases (JAK); the involvement of the Src and Abl tyrosine kinases as well as EGFR have also been reported [3]. Tyrosine phosphorylation of STAT3 is followed by dimerization

\section{C) Biomed Central}


through phosphotyrosine-SH2 domain interaction; activated STAT3 enters the nucleus where it stimulates the transcription of its targets, including Cyclin-D1, Survivin, Vegf, C-Myc, Bcl-xL, and Bcl2 [4,5].

STAT3 is a key regulator of cell survival and proliferation [6]. Its constitutive activation has been observed in many human tumors, including colon, breast, lung, pancreas and prostate cancers, melanoma, head and neck squamous carcinoma, multiple myeloma, mantle cell lymphoma, and glioma $[7,8]$. However, in certain cell types such as PTEN-deficient glioblastoma, STAT3 can become a tumor suppressor $[9,10]$.

STAT1 is another member of the STAT family. It is activated mainly by IFNs $\alpha$ and $\gamma$, and plays a major role as a pro-inflammatory, anti-pathogen and anti-proliferative factor $[11,12]$. Its biological function is thus mostly antagonistic to that of STAT3. Despite their 50\% amino acid sequence homology [13], STAT1 and STAT3 are structurally very similar; yet some important differences have been noted in their DBD sequences [14]. Despite its major role as a tumor antagonist, STAT1 can also have functions in cancer cells, as documented in mouse leukemia [15].

Inhibition of STAT3 in tumor cells in which it is constitutively activated leads to cell death [16-18]. This is achieved using either non-specific inhibitors such as curcumin, which also inhibits other transcription factors (NF$\kappa \mathrm{B})$, or inhibitors specifically designed to inhibit STAT3 through non-covalent binding to the $\mathrm{SH} 2$ domain, such as Stattic [19] or STA-21 [20]. Interestingly, these compounds have little effect in cells in which STAT3 is not activated, pointing to STAT3 as a highly valid target to focus on for the design of anti-cancer compounds. However, such compounds are still poorly developed.

TFs activate transcription of their target genes by binding to distinct short DNA consensus motifs. Decoy oligonucleotides (dODNs) containing these consensus motifs can bind the DNA binding domains (DBD) of the TFs and block their activity. dODNs [21] and hairpin dODNs (hpdODNs) [22] have been shown to induce the death of cells in which STAT3 is activated, suggesting that the DBD is another potential target for specific inhibitory compounds. Similarly to double-stranded oligonucleotides that are used to detect active dimers in electrophoretic migration shift assays, STAT3 hpdODNs interact with activated, dimeric STAT3. This interaction impairs the binding of the dimer to importins, resulting in the sequestration of STAT3 in the cytoplasm [23].

Yet, because of the high degree of similarity between STAT3 and STAT1 consensus DNA binding sites, STAT1 competes with activated STAT3 for dODN binding in interferon (IFN) $\gamma$-treated cells [22,23], thereby preventing inhibition of active STAT3. Under such conditions the dODN loses its ability to block cell proliferation. In addition, since STAT1 plays a key role in cell death processes [24,25], including caspases expression [26,27] and cooperation with p53 function $[28,29]$, its inhibition by the dODN prevents cell death. Finally, IFN $\gamma$ being a cell death inducer in several cell types [22,30-32], it is important to design reagents that do not interfere with STAT1, one of its key effectors. Thus, in order to elaborate target-specific anti-cancer compounds, the specificity of hpdODNs to STAT3 needs to be enhanced. It should be noted, however, that in certain cellular contexts STAT1 has been found to be a tumor promoter [15].

The difficulty in designing dODNs recognized by STAT3 but not STAT1 lies in the striking similarity of the consensus DNA sequences of the two TFs [33], in spite of their different cellular functions. Nevertheless, early studies on STAT3/STAT1-discriminating DNA motifs established some sequence preferences that differentiate these TFs [34-39], suggesting possibilities for designing STAT3/ STAT1-discriminating dODNs. The notion that discrete nucleotide modifications in target DNA sequences might alter their recognition by closely related TFs is supported by the observation that a single nucleotide change in the $\kappa \mathrm{B}$ consensus motif modified NF- $\kappa \mathrm{B}$ subunit specificity [40]. Furthermore, DNA recognition by proteins relies in part on DNA shape, known to deviate from the ideal Bconformation. The nature of the nucleotides in the sequence influences conformation and dynamics: for instance, $\mathrm{dG}: \mathrm{dC}$ stretches confer rigidity [41], pyrimidine/ purine steps (particularly $\mathrm{T} / \mathrm{dA}$ steps) confer flexibility and may also introduce kinks $[42,43]$, and dA:T stretches can have complex configurations [41]. The coordinates from available crystal structures of both STAT1 [44] and STAT3 [45] (in complex with their DNA consensus sequence) were used to analyze their 3D structure using the UCSF Chimera program [46]. Based on the differences found, new hpdODNs were designed and tested for their STAT3/STAT1 discrimination ability by measuring SW480 colon carcinoma cell death and absence of inhibition of STAT1-dependent IFN $\gamma$-induced cell death. SW480 cells offer a relevant model since these cells show constitutive activation of STAT3, which is essential for their survival, and they are susceptible to IFN $\gamma$-induced cell death, which is a STAT1-dependent process. The newly designed hpdODNs were also compared for their relative binding capacity to STAT1 and STAT3 by performing in-cell pull-downs, and for their ability to prevent nuclear transfer using immunofluorescence.

\section{Results}

Striking similarities in the interactions of STAT1 and STAT3 with their consensus DNA sequence

Comparison of the 3D structures of STAT1 and STAT3 in complex with their oligonucleotide duplexes featuring 
a consensus DNA sequence using the Chimera program showed that they are highly similar (Figure 1A), with an overall root mean square deviation of $0.63 \AA$ between 317 atom pairs of the backbone. To focus our study on the interaction of the STAT1 and STAT3 DBDs with their consensus DNA sequence, only the amino acids in close contact with the DNA strands were examined. This revealed the striking similarity of STAT1 and STAT3 DNA-interacting amino acids (Figures 1B and 1C). Several differences were noted, however, including:
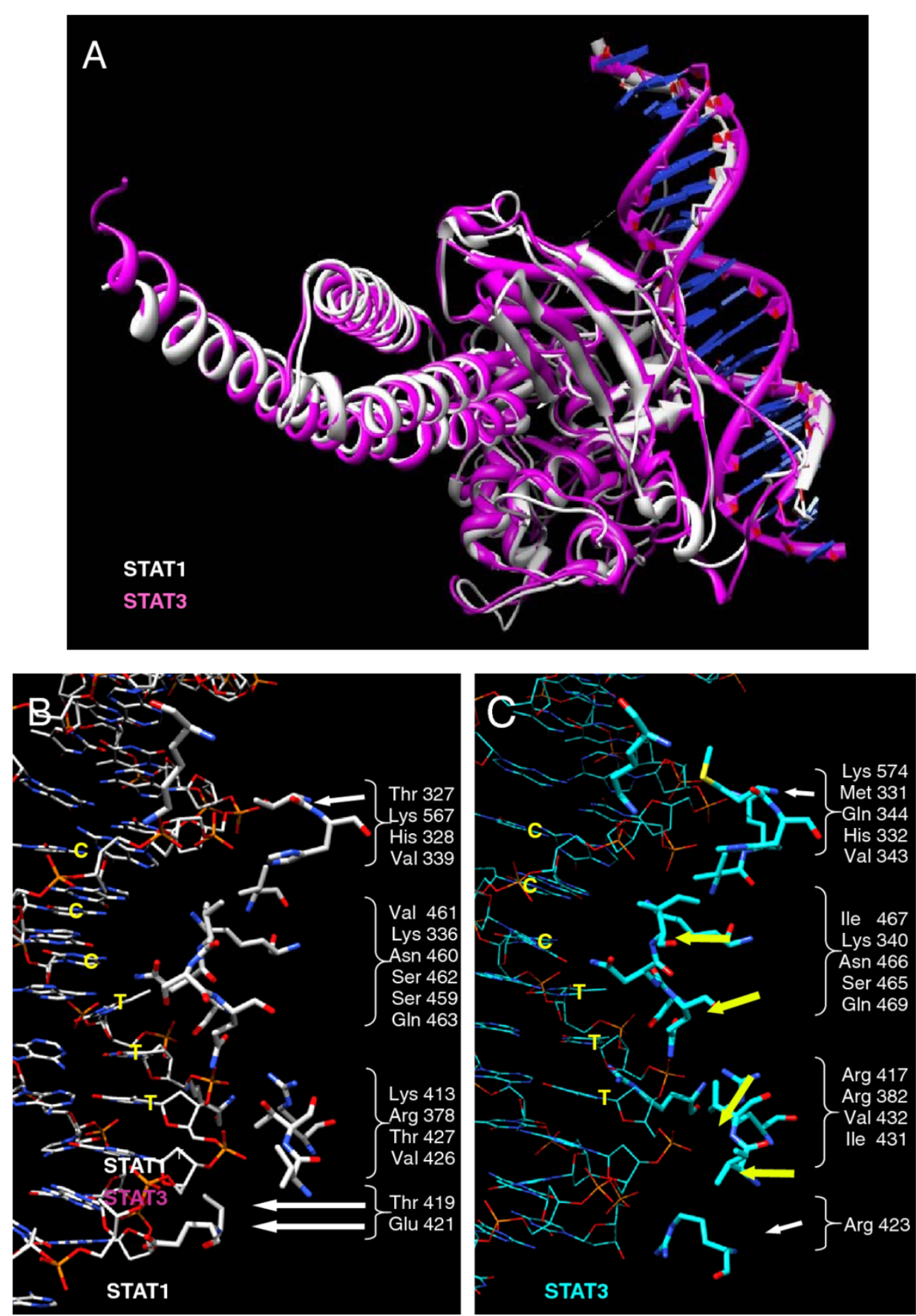

Figure 1 3D comparison of STAT1 and STAT3 and of their DNA binding region. A: View of the superimposed 3D structure of STAT1 and STAT3 monomers created with the Chimera program, showing the DNA binding region. $\mathbf{B}$ and $\mathbf{C}$ : close-up views of the DNA binding regions of STAT1 (B) and STAT3 (C). Amino acids shown are those within a $5 \AA$ distance from DNA; note that Thr 327 did not appear using this selection and was added. Arrows point to amino acids of STAT1 and STAT3 interacting differently with DNA. 
i) Glu 421, unique to STAT1, and located within direct H-bond distance from G 1017 [44], G 2002 and C 1018 (the latter possibly mediated by water) (Figures $1 \mathrm{~B}$ and $1 \mathrm{C}$; detailed view in Figure 2; see Table 1 for a list of $\mathrm{H}$ bonds and Additional file 1: Table S1 for a list of the interactions of STAT1 and STAT3 with DNA); ii) the peptide backbone of a polar residue of STAT1, Thr 327, and of a hydrophobic residue of STAT3, Met 331, establish H bonds with C 1009 and C 1010 (Figure 1B); iii) a polar amino acid, Thr 419 for STAT1, and a charged amino acid, Arg 423 for STAT3, are identically positioned, facing the backbone of nucleotide 1018 (Figure 1B). To obtain STAT3/STAT1 discriminating sequences, we chose to design hpdODNs, by modifying the original consensus sequences at the specific positions where interactions with STAT1 and STAT3 were found to differ (the consensus sequences and hpdODNs are depicted in Figure 3: for convenience we use the same numbering as in reference [45]).

\section{Nucleotide substitutions provide a hairpin decoy oligonucleotide which can discriminate between STAT1 and STAT3, inhibiting STAT3 in IFN $\gamma$-treated cells}

As previously shown [22,23], the consensus-carrying hpdODN A can efficiently induce the death of cells of the SW480 line (Figure 4A and 4B); but it also inhibits STAT1, thus blocking the STAT1-dependent IFN $\gamma$ induced mortality of these cells (Figure 4A, B and 4C) as previously shown [22,23]. hpdODN B was designed by replacing three base pairs in hpdODN A. T replaced dC in position 1003, dC replaced dG in 1011, and dG replaced $\mathrm{dC}$ in position 1017 (Figure 3). In the same assay, hpdODN B was found to efficiently induce SW480 cell

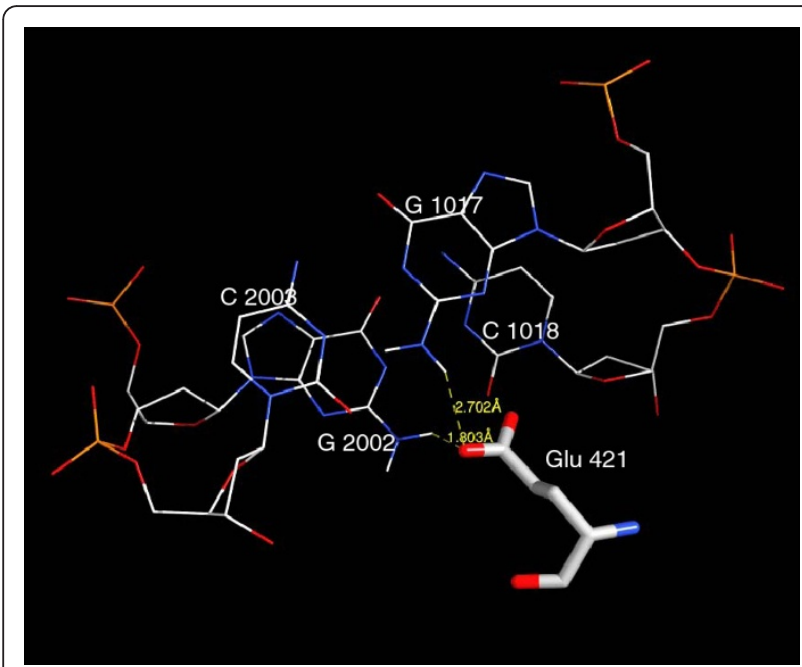

Figure 2 Location of Glu 421 (STAT1). A detailed view of the close interactions of Glu 421 of STAT1 with C 1018, G 1017 and G 2002 in the minor groove of the double helix. death but was devoid of any action on IFN $\gamma$-induced cell death (Figure 4A and 4B), indicating a preference for STAT3 over STAT1. Features of hpdODN B consist in a stretch of pyrimidines spanning nucleotides 1005 to 1012, a d(TA) step (1003/1004) and a d(TG) step (1016/1017). To analyze the possible effect of only one change in the sequence of hpdODN A, hpdODN C was designed by replacing $\mathrm{dG}$ with $\mathrm{dC}$ in position 1011 (Figure 3). The killing efficiency of HpdODN C was lower than those of hpdODN A $[22,23]$ and hpdODN B, but in contrast with the latter, it showed a capacity to compete with IFN $\gamma$ induced mortality, suggesting that it interacts with STAT1 (Figure 4A and 4B). Next, by placing dG in 1003, dC in 1004, dC in 1011 and dG in 1017 we obtained hpdODN D (Figure 3), which corresponded with a sequence with a marked preference for STAT1 as previously shown by others using a reporter assay [47]. hpdODN D did not induce SW480 cell mortality, but prevented IFN $\gamma$-induced killing (Figure 4A and 4B). Finally, hpdODN E, containing a mutated STAT3 binding site (Figure 3) did not induce cell death and did not compete with IFN $\gamma$-induced cell death (Figure 4A and 4B). A comparison of the different hpdODNs' IFN $\gamma$-independent cell killing efficiency (taking the killing efficiency of hpdODN A as the 100\% standard) showed that hpdODN B was twice as efficient as hpdODN A (Figure 4C) and that the control mutated hpdODN E had no effect on cell death (Figure $4 \mathrm{C}$ ), as previously published [22,23].

The new STAT3-specific hpdODN B inhibits STAT3 but not STAT1 phosphorylation and inhibits cyclin D1 but not IRF1 expression

To detect the effect of the hpdODNs on STAT3 phosphorylation, IL-6-treated $(50 \mathrm{ng} / \mathrm{ml}) \mathrm{SW} 480$ cells were used. In cells treated with hpdODN B and hpdODN A for $16 \mathrm{~h}$, STAT3 phosphorylation was suppressed (Figure 4E, lanes 2 and 3); the expression of cyclin D1 and of STAT3 itself (STAT3 being its own target) were considerably diminished (Figure 4E, lanes 2 and 3 ), in agreement with previous observations [22,23]. When cells were treated for $4 \mathrm{~h}$ with hpdODNs A and B, phospho-STAT3 was reduced without effect on STAT3 (Figure 4F); the control mutated hpdODN E had no effect (Figures 4E and 4F, lane 4). To confirm that hpdODN B was preferentially inhibiting STAT3 in SW480 cells, the induction of the STAT1-dependent IFN $\gamma$ target IRF1 was studied. In cells treated with IFN $\gamma(50 \mathrm{ng} / \mathrm{ml}$ for 16 h), both phosphorylation of STAT1 and expression of IRF1 increased (Figure 4D, lanes 1 and 2). Treatment with hpdODN A, but not hpdODN B, strongly reduced IRF1 expression (Figure 4D, lanes 3 and 4). In IFN $\gamma$ treated cells, the addition of hpdODN A reduced IFN $\gamma$ induced IRF1 expression (lane 5) whereas the addition of hpdODN B did not (lane 6). Interestingly, STAT1 
Table 1 Hydrogen bonds involved in the interaction of STAT1 and STAT3 with DNA

\begin{tabular}{|c|c|c|c|}
\hline \multicolumn{2}{|c|}{ STAT1 } & \multicolumn{2}{|c|}{ STAT3 } \\
\hline Nucleotide involved & Residue(s) involved & Nucleotide involved & Residue(s) involved \\
\hline T 1005 & Thr 427 (BB/Phos.) & T 1005 & Val 432 (BB/Phos.) \\
\hline \multirow[t]{5}{*}{ T 1006} & Lys 413 (BB/Phos.) & T 1006 & Ser 465 (SC/Phos.) \\
\hline & Ser 459 (BB/Phos.) & T 1006 & Arg 382 (SC/Phos.) \\
\hline & Arg 378 (BB/Phos.) & T 1006 & Gln 469 (SC/Phos.) \\
\hline & Ser 459 (BB/Phos.) & Т 1006 & Ser 465 (SC/Phos.) \\
\hline & Gln 463 (BB/Phos.) & & \\
\hline \multirow[t]{4}{*}{ T 1007} & Asn 460 (BB/Base) & T 1007 & Asn 466 ND2(SC/Base) \\
\hline & & T 1007 & Arg 417 NH1 (SC/Phos.) \\
\hline & & C 1008 & Gln 344 NE2 (SC/Phos.) \\
\hline & & C 1009 & Gln 344 N (BB/Phos.) \\
\hline \multirow[t]{2}{*}{ C 1010} & His 328 (SC/Phos.) & C 1010 & Lys 340 (SC/Phos.) \\
\hline & & C 1010 & His 332 (SC/Phos.) \\
\hline G 1011 & Lys 567(SC/Phos.) & & \\
\hline Т 1012 & Asn 460 (SC/Base) & T 1012 & Asn 466 (SC/Base) \\
\hline G 1017 & Glu 421 (SC/Base) & & \\
\hline \multirow[t]{2}{*}{ C 1018} & Thr 419 (BB/Phos.) & & \\
\hline & Thr 419 (BB/Phos.) & & \\
\hline \multirow[t]{2}{*}{ G 2002} & Glu 421 (SC/Base) & & \\
\hline & Glu 421 (SC/Base) & & \\
\hline T 2005 & Thr 427 (BB/Phos.) & T 2005 & Val 432 (BB/Phos.) \\
\hline \multirow[t]{4}{*}{ T 2006} & Lys 413 (SC/Phos.) & T 2006 & Ser 465 (SC/Phos.) \\
\hline & Arg 378 (SC/Phos.) & T 2006 & Arg 382 (SC/Phos.) \\
\hline & Ser 459 (SC/Phos.) & T 2006 & Ser 465 (SC/Phos.) \\
\hline & Arg 378 (SC/Phos.) & T 2006 & Gln 469 (SC/Phos.) \\
\hline \multirow[t]{2}{*}{ T 2007} & Asn 460 (SC/Base) & T 2007 & Asn 466 (SC/Base) \\
\hline & Lys 413 (SC/BB) & T 2007 & Arg 417 (SC/Phos.) \\
\hline \multirow[t]{2}{*}{ A 2008} & Asn 460 (SC/Base) & A 2008 & Gln 344 (SC/Phos.) \\
\hline & & C 2009 & Gln 344 (BB/Phos.) \\
\hline \multirow[t]{3}{*}{ G 2010} & His 328 (SC/Phos.) & G 2010 & Lys 340 (SC/Base) \\
\hline & & G 2010 & His 332 (SC/Phos.) \\
\hline & & G 2010 & Lys 340 (SC/Phos.) \\
\hline G 2011 & Lys 567 (BB/Phos.) & & \\
\hline G 2012 & Asn 460 (SC/Base) & G 2012 & Asn 466 (SC/Base) \\
\hline A 2013 & Asn 460 (SC/Base) & & \\
\hline G 2018 & Glu 421 (SC/Phos.) & & \\
\hline
\end{tabular}

The nucleotides that are involved in binding with STAT1 or STAT3 are listed. For clarity, the numbering used is the same as in reference [45] (see Figure 3). The following abbreviations were used for the atoms involved in hydrogen bonding: phospho-diester (Phos.) or base moiety (Base) for the nucleotides. BB stands for backbone and SC for side-chain for the proteins.

phosphorylation on tyrosine was inhibited following treatment with hpdODN A (lane 5) but not with hpdODN B (lane 6). These data indicate that under these experimental conditions hpdODN B does not inhibit STAT1.

\section{Biotinylated hpdODN B interacts preferentially with STAT3}

Binding of STAT3 and STAT1 to hpdODNs has previously been analyzed directly within cells using biotinylated versions of the different hpdODNs [23]. To compare hpdODNs A and B, cells were treated, or not, with IFN $\gamma$, transfected with biotinylated hpdODNs, and pull-downs were performed. The pull-down efficiencies of hpdODN A (Figure 5, lanes 1 and 4) and B (Figure 5, lanes 3 and 6) for STAT1 and STAT3 were very different. Indeed, compared with hpdODN A, hpdODN B brought down STAT3 very efficiently, but not STAT1 (Figure 5, lanes 1 and 3), even in IFN $\gamma(50 \mathrm{ng} / \mathrm{ml}$ ) -treated cells (Figure 5, compare lanes 4 and 6). Furthermore, compared 


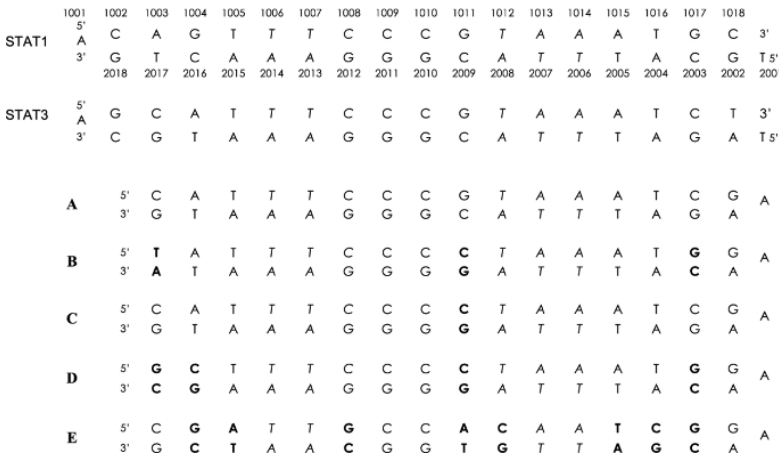

Figure 3 Sequences used in this study. The first two sequences depicted are the ones used in the X-ray crystallographic studies of STAT3 [45] and STAT1 [44], based on a STAT3 consensus sequence derived from the c-fos gene promoter [34,35] and a STAT1 consensus sequence derived from the IRF-1 gene promoter $[34,35,59]$. For clarity, the same numbering (shown here above the STAT1 consensus sequence) was used for all sequences throughout the study. Bases of the consensus sequence are in italic. HpdODN A contains the optimal STAT3 consensus sequence based on the STAT3 binding consensus [34], used in [21,22]. Compared with hpdODN A, hpdODN B is modified at three locations: 1003, 1011 and 1017; hpdODN C is modified at 1011 only; and hpdODN D is modified at 1003, 1004, 1011 and 1017. HpdODN E is a mutated negative control which is modified at 1004, 1005, 1008, 1011, 1012, 1015, 1016 and 1017. Modified bases are in bold.

with hpdODN A, hpdODN D, shown to interact preferentially with STAT1, was more efficient in pulling down STAT1 than STAT3 (compare lanes 8 and 9 with lanes 1 and 4). Finally, hpdODN E, a control hpdODN with mutations in the binding consensus, did not bring down either STAT1 or STAT3 (Figure 5, lane 7).

\section{The new hpdODN B prevents the constitutive nuclear location of STAT3 in SW480 cells, but not that of IFN $\gamma$ - activated STAT1}

HpdODNs A and B were further compared for their ability to prevent the nuclear translocation of STAT3 and STAT1 in SW480 cells (treated or not with IFN $\gamma$ ) using immunofluorescence. Treatment of the cells with hpdODN A prevented the nuclear translocation of both STAT3 and STAT1 (for STAT1 nuclear translocation, IFN $\gamma$-treated cells were used) (Figure 6), as previously shown $[22,23]$. Treatment with hpdODN B prevented the nuclear translocation of STAT3 only, and not that of IFN $\gamma$-activated STAT1 (Figure 6, columns 1 and 4, see arrows), confirming its discriminative capacity. Notably, the control mutated hpdODN E had no effect on the subcellular location of either STAT3 or STAT1, which both remained nuclear (Figure 6, columns 2 and 5).

\section{Discussion}

A new hairpin decoy oligonucleotide (hpdODN) carrying STAT3's DNA binding consensus sequence was designed following 3D analysis of protein/DNA interaction and shown to induce the death of STAT3-dependent tumor cells without interfering with STAT1, a key effector of cell death. In this paper, 3D structural analyses of the protein/DNA interaction of STAT1 and STAT3 demonstrated their high similarity, confirming previous reports [13]. These 3D analyses served as a basis for the design of new sequences with base substitutions. The new sequences were tested for their ability to induce cell death in an IFN $\gamma$-sensitive [22,23,48-51], active-STAT3-dependent colon carcinoma cell line [22]. This enabled the design of the STAT3-specific hpdODN labeled here as hpdODN B. The ability of hpdODN B to discriminate between STAT1 and STAT3 was assessed by: i) its ability to kill cells without interfering with IFN $\gamma$-induced cell death; ii) its ability to inhibit STAT3 targets, including cyclin D1, iii) the absence of inhibition of IFN $\gamma$-induced STAT1 phosphorylation and IRF1 expression, iv) its lack of interaction with STAT1 in pull-down assays and iv) its inability to inhibit IFN $\gamma$ induced STAT1 nuclear location. Indeed, hpdODN A treatment, but not hpdODN B treatment, reduced STAT1 phosphorylation, probably by impairing nucleocytoplasmic shuttling as previously suggested [22,23]. Nevertheless, despite its ability to discriminate between STAT1 and STAT3, hpdODN B probably has a residual affinity for STAT1, as shown by low detection of STAT1 in pull-down assays and the fact that cell death induction by hpdODN B and IFN $\gamma$ are not additive.

The STAT3/STAT1-discriminating hpdODN was obtained by replacing key nucleotides that $3 \mathrm{D}$ analyses had shown to be in the vicinity of amino acids of the DBD that distinguish the two STATs; the similarity of their DNA consensus sequences, despite their different functions, has been recognized for some time [33]. Examination of the nucleotide modifications that led to STAT1/STAT3-discriminating hpdODN B showed that they are compatible with previous in vitro DNA-binding studies, such as the preference for $\mathrm{T}$ at 1003 and 1005, $\mathrm{dC}$ at 1010 and $\mathrm{dA}$ at 1015 of STAT3 [36-38]. The fact that $\mathrm{T}$ at 1003 does not favor STAT1 binding is also in agreement with the earlier suggestion that "selection for a dG:dC base pair at position 7 (here: 1017) is likely to involve Glu 421 (of STAT1) which can accept hydrogen bonds from guanine in the minor groove" [44]. This has also been noted by others [39]. Finally, altered recognition by a TF following single nucleotide changes has been previously shown, for instance with NF- $\kappa$ B subunit recognition of $\kappa \mathrm{B}$ [40]. One notable property of the hpdODN B is its dissymmetry. A symmetric version (with $\mathrm{dA}$ replacing $\mathrm{dG}$ in 1017) was tested and is apparently not different from hpdODN B (not shown). Intriguingly, although the preference of hpdODN D for STAT1 was expected from previous data showing its 


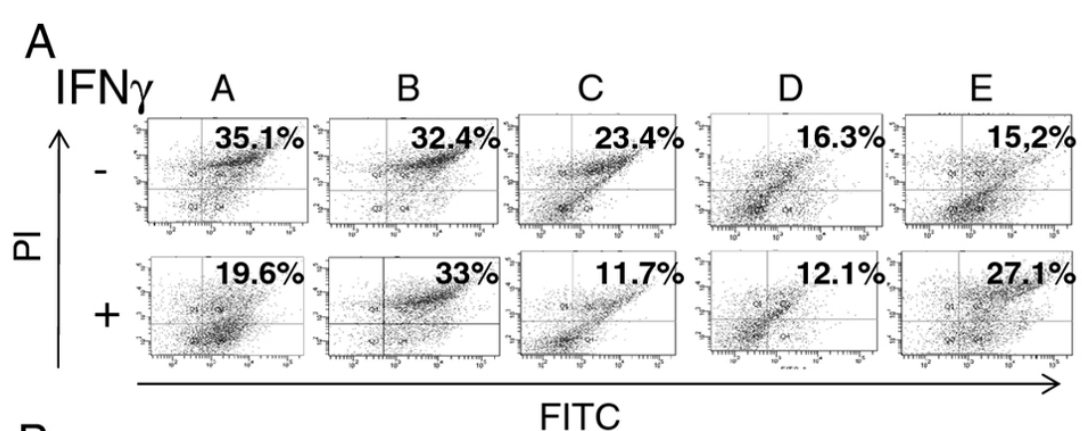

B

FITC
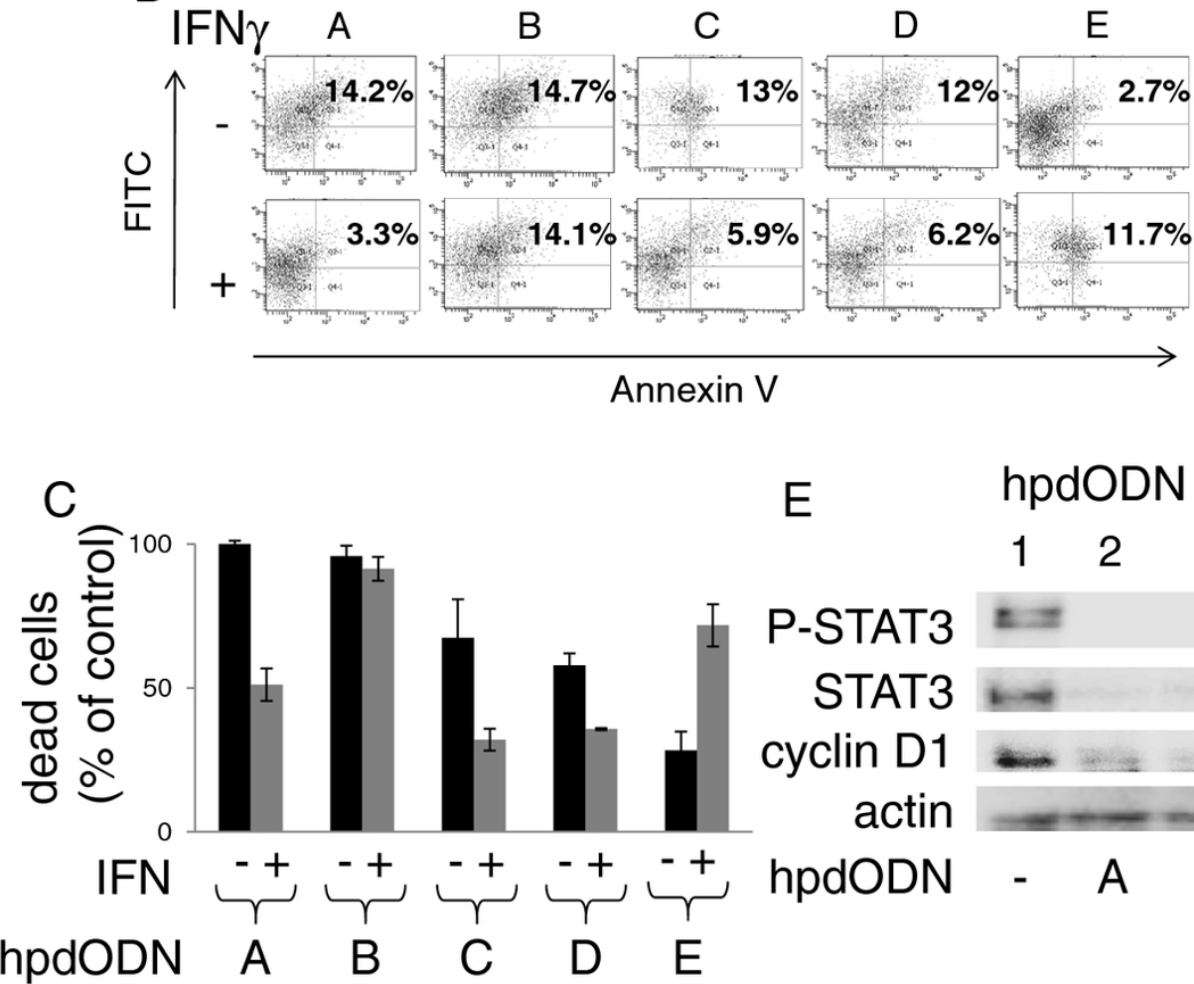

$E$

hpdODN $16 \mathrm{~h}$

D

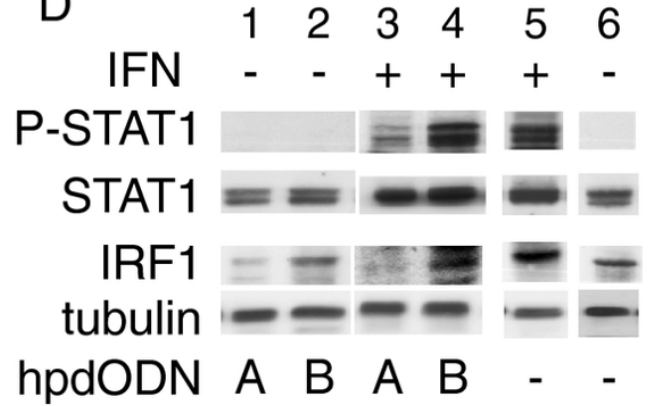

$\mathrm{F}$

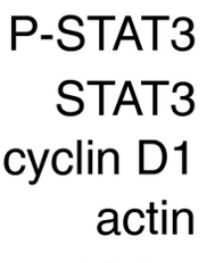

actin

\section{$\begin{array}{llll}1 & 2 & 3 & 4\end{array}$}

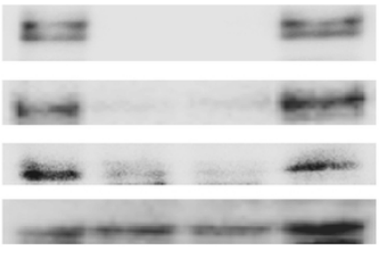

A B E

hpdODN $4 \mathrm{~h}$
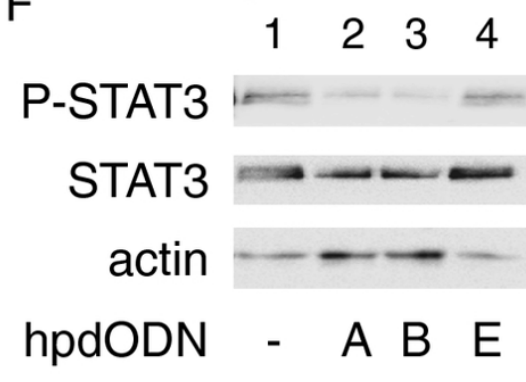

Figure 4 Comparative cell death induction efficiency of the different hpdODNs in the presence or not of IFN $\gamma$ in the SW480 cell line. The efficiencies of hpdODN A, B, C D and $\mathrm{E}$ in inducing cell death were compared, in the presence (+) or not (-) of IFN $\gamma$. A. Cell death, measured by determining propidium iodide (PI) labeling, induced by hpdODNs A to E (2 $\mu \mathrm{g} / \mathrm{ml})$ alone (-) or in the presence of IFN (50 $\mathrm{ng} / \mathrm{ml}$ 16 h) (+). B. Cell death, measured with flow cytometry for annexin V, induced by hpdODNs A to $E(2 \mu \mathrm{g} / \mathrm{ml})$ alone $(-)$, or in the presence of IFN $(50 \mathrm{ng} / \mathrm{ml} 16$ h) (+). In A and B, FITC denotes the detection of cells having incorporated the fluorescein-labelled hpdODNs. Note that experimental conditions were such that cell death was not maximal, to allow detection of differences between experiments. Experiments were repeated at least three times, a typical result is shown. C: Histograms from several experiments (at least three per condition) using hpdODNs A to E, obtained by computing PI incorporation and annexin V labelling data (such as those shown in Figures 4A and B). To facilitate comparison, data are presented as\% of the control. D: Western blotting showing the phosphorylation of STAT1 on tyrosine ("P-STAT1") and expression level of 
IRF1 in the absence (lanes 1, 3 and 4) or presence of IFN $(50 \mathrm{ng} / \mathrm{ml}, 16 \mathrm{~h}$ ) (lanes 2, 5 and 6) and in the presence or not of the indicated hpdODNs $(2 \mu \mathrm{g} / \mathrm{ml})$. Anti-phospho-STAT1, anti-STAT1, anti-IRF1 and anti-tubulin antibodies were used. Experiments performed with the mutated control hpdODN E showed no effect (not shown). E: Western blotting showing the inhibition of STAT3 phosphorylation and the reduced expression of cyclin D1 and STAT3 following treatment with hpdODN A (lane 2) or hpdODN B (lane 3), or the mutated hpdODN E (lane 4) (2 $\mu \mathrm{g} / \mathrm{ml}$ for $16 \mathrm{~h}$ ). F: Western blotting showing the inhibition of STAT3 phosphorylation following treatment with hpdODN A (lane 2) or hpdODN B (lane 3), or the mutated hpdODN E (lane 4) $(2 \mu \mathrm{g} / \mathrm{ml}$ for $4 \mathrm{~h}$ ). Experiments were repeated at least three times, one representative experiment is shown.

STAT1-specific binding [47], its basis is not clear and may rest upon properties beyond nucleotide sequence such as DNA shape. The shape and flexibility of DNA strands are known to be influenced by their nucleotide content; here the 8-pyrimidine stretch in hpdODN B may confer a higher flexibility than hpdODN A and may account for a differential interaction with STAT3 Arg 423 and STAT1 Glu 421. In fact, the molecular dynamics studies which describe a scissor-like molecular movement upon DNA binding for STAT3, but not for STAT1 [13] suggest that the flexibility of the DNA target may play a role in binding and therefore underly the preference of hpdODN B for STAT3. It may also account for the greater sensitivity of STAT3 to an intact palindromic structure compared to STAT1, as previously stated [38]. Protein binding itself can affect DNA bending, as shown with the high-affinity target of the papillomavirus E2 [52]. Nevertheless, despite its efficiency, the precise mechanism whereby the hpdODN B discriminates between STAT1 and STAT3 in cells is not understood. Changes in DNA shape may play a role in the preferential recognition of hpdODN B by STAT3; co-factors may also be involved in DNA recognition by STAT3, and might associate more efficiently when hpdODN B is used. The process might also be more complex than mere differential DNA binding: STAT1 and STAT3 are reciprocally regulated $[2,53-55]$ and the relative abundance of their active forms may itself play a key role in biological responses, as previously discussed [56]. Another level of complexity arises from the fact that in cells in which STAT3 has been suppressed, IFN $\gamma$-activated STAT1 induces the expression of mitogenic STAT3-targets $[57,58]$. Furthermore, STAT1 and STAT3 form heterodimers, whose function has not been elucidated to date. In this respect, quantification of the relative amounts of STAT1 and STAT3 bound to the hpdODNs A and B may help understand the complex interaction of these TFs. Preliminary experiments that are underway suggest a difference in heterodimer content. Therefore, it is possible that hpdODN B functions in cells by tilting the active STAT1/active STAT3 balance toward STAT1, thereby inducing cell death.

\section{Conclusions}

By combining 3D molecular interaction analysis and direct screening in cells, this work allowed the design of an hpdODN that can selectively inhibit STAT3 but not STAT1. The efficacy and potential of this approach resides in the direct testing of modified hpdODNs in cells, analyzing processes that depend on STAT3 (cell

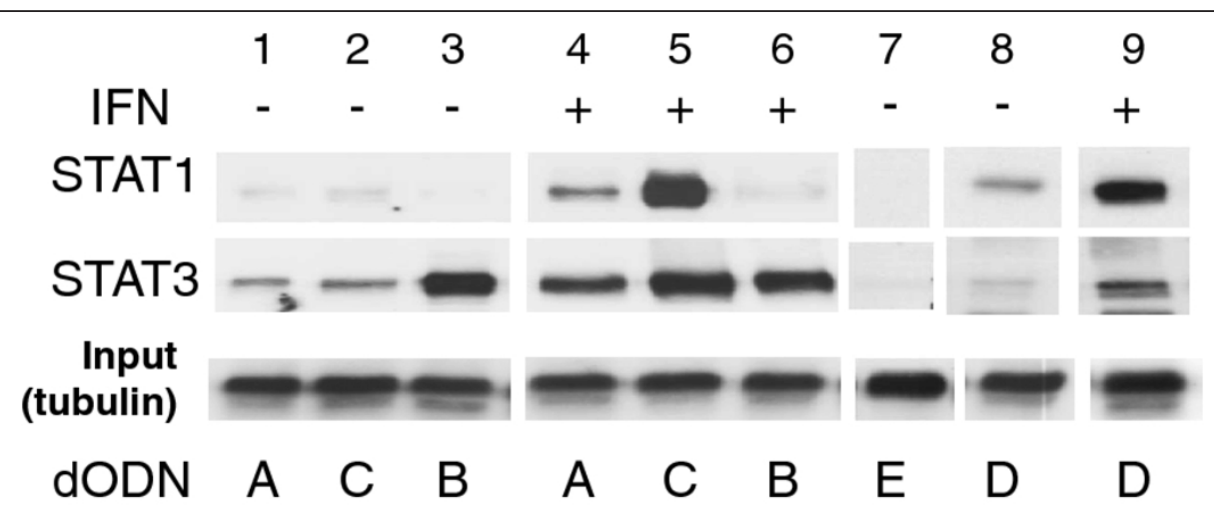

Figure 5 Specific binding of hpdODN B to STAT3 detected by in-cell pull-down. Western-blotting of STAT1 and STAT3 bound to biotinylated hpdODNs after transfection and in-cell pull-down with avidine-coated beads. Cells were either not treated (-) (lanes 1, 2, 3 and 7, 8) or treated (+) (lanes 4, 5, 6 and 9) with IFN $(50 \mathrm{ng} / \mathrm{ml}, 16 \mathrm{~h}$ ), and hpdODN A (lanes 1 and 4), hpdODN B (lanes 3 and 6), hpdODN C (lanes 2 and 5) hpdODN D (lanes 8 and 9) and hpdODN E (lane 7) (hpdODNs were at $1 \mu \mathrm{g} / \mathrm{ml}$ for $16 \mathrm{~h}$ ). Following cell lysis, protein concentration was measured, identical amounts of protein per sample $(250 \mu \mathrm{g})$ were added; anti-tubulin western blotting from aliquots of the extracts was performed to show identical loading. Binding experiments were repeated at least three times. All lanes are part of the same blot (identical exposure time) and have been separated for clarity. 


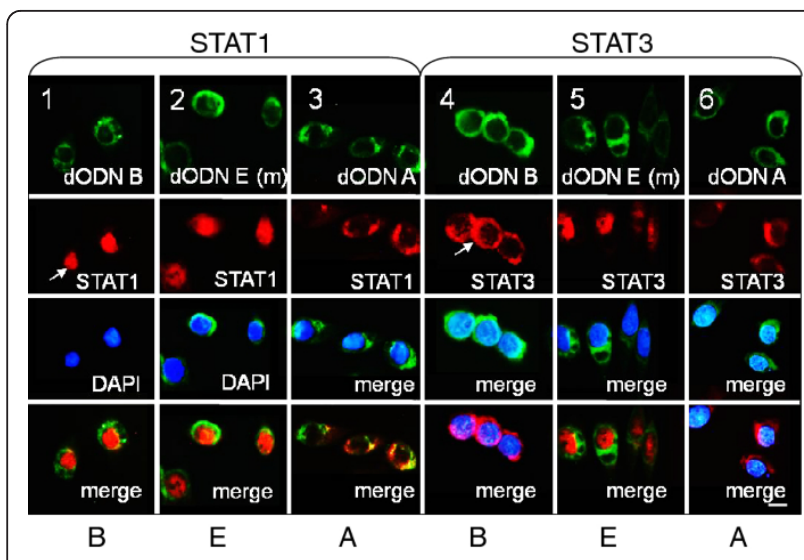

Figure 6 Differential inhibition of STAT1 and STAT3 nuclear transfer by hpdODN B. Immunofluorescence analysis of STAT1 and STAT3 nuclear transfer in SW480 cells incubated with fluoresceinlabelled hpdODNs A, B and E (green). STAT1 nuclear transfer was induced by treating cells with IFNy (50 ng/ml, $16 \mathrm{~h}$ ) (columns 1, 2 and 3). Columns 3 and 6: hpdODN A; columns 1 and 4: hpdODN B; columns 2 and 5: hpdODN E (mutated control). All hpdODNs were at $1 \mu \mathrm{g} / \mathrm{ml}$. STAT1 and STAT3 were detected using specific antibody and a second rhodamine-coupled antibody (red), nuclei (blue) were stained using DAPI and detected under UV light; the different images were merged to examine the subcellular location of the fluorescein-tagged hpdODNs (green) and STAT1 or STAT3 (red). Arrows point to nuclear STAT1 and cytoplasmic STAT3 in IFN $\gamma$ treated and hpdODN B-treated cells. Size bar: $10 \mu \mathrm{m}$. One representative experiment out of at least three is shown.

survival) or STAT1 (IFN $\gamma$-mediated death). These hpdODNs represent a basis for elaborating STAT3 DBD-specific low molecular weight compounds with anti-cancer properties.

\section{Material and methods}

Computer analysis of STAT3 and STAT1

The PDB (Protein Data Base) files for STAT1 (1BF5) [44] and STAT3 (1BG1) [45] were downloaded and analyzed using Chimera [46]. The STAT1 and STAT3 crystals used in the X-ray diffraction studies were proteins complexed with oligonucleotide duplexes featuring a consensus DNA sequence [34,35,59] (see Figure 3). To compare the STAT1 and STAT3 DBDs in a complex with their DNA consensus sequences, the missing complementary strand of the STAT3-bound oligonucleotide [45] was reconstructed through crystal symmetry operations.

\section{Decoy oligonucleotides}

The STAT3 decoy ODNs used were RHN- $\left(\mathrm{CH}_{2}\right)_{6}$-CATTTCCCGTAAATCGAAGATT TACGGGAAATG- $\left(\mathrm{CH}_{2}\right)_{6}$-NHR (STAT3 decoy hpdODN), derived from the serum-inducible element of the human c-fos promoter [34] (hpdODN A) and previously used in the lab $[22,23]$.
RHN- $\left(\mathrm{CH}_{2}\right)_{6}$-TATTTCCCCTAAATGGAACATT TAGGGGAAATA- $\left(\mathrm{CH}_{2}\right)_{6}$-NHR (hpdODN B); RHN$\left(\mathrm{CH}_{2}\right)_{6}$-CATTTCCCCTAAATCGAAGATTTAGGGGAAATG- $\left(\mathrm{CH}_{2}\right)_{6}$-NHR (hpdODN C); RHN- $\left(\mathrm{CH}_{2}\right)_{6-}$ GCTTTCCCCTAAATGGAACATTTAGGGGAAAGC$\left(\mathrm{CH}_{2}\right)_{6}$-NHR (hpdODN D); and the following mutated hpdODN as a negative control:

RHN- $\left(\mathrm{CH}_{2}\right)_{6}$-CGATTGCCACAATCGGAACGATTG TGGCAATCG- $\left(\mathrm{CH}_{2}\right)_{6}$-NHR (hpdODN E) (where R was either: hydrogen, fluorescein or biotin). The addition of fluorescein or biotin, followed by high-performance liquid chromatography, were carried out by the manufacturer (Eurogentec, Seraing, Belgium) using in-house protocols. The hairpin sequence GAA, previously shown to confer stability and nuclease resistance [60,61], was included in the dODNs. In the hpdODNs, the hairpin motif was built and incorporated in the X-ray structure using the BCE (Biomolecular Chain Elasticity) approach [62]; this showed that the hairpin did not interfere with the DBD-DNA interaction (not shown).

\section{Cell culture and reagents}

SW480 (colon adenocarcinoma) cells were grown in DMEM (Invitrogen, Lifetechnologies, Cergy-Pontoise, France), supplemented with 10\% FCS (Lonza, LevalloisPerret, France), $100 \mathrm{U} / \mathrm{ml}$ penicillin, $10 \mu \mathrm{g} / \mathrm{ml}$ streptomycin (GibcoBRL), $1 \mathrm{mM}$ sodium pyruvate (GibcoBRL), MEM vitamins (100x, Invitrogen) and $5 \mu \mathrm{g} / \mathrm{ml}$ plasmocin (Cayla InvivoGen, Toulouse, France). Sodium orthovanadate $(100 \mu \mathrm{M})$ was from Fischer (Illkirch, France). Interferon $\gamma(50 \mathrm{ng} / \mathrm{ml})$ was from Promocell $(\mathrm{GmbH}$, Heidelberg, Germany) or Sigma-Aldrich (Montigny-leBretonneux, France).

\section{Transfections}

Cells were grown in 4-well plates to a density of $0.25 \times$ $10^{6}$ cells $/ \mathrm{ml}$. When the cells reached $50-60 \%$ confluence, they were transfected with the different STAT3 hpdODNs or the control hpdODN $(2 \mu \mathrm{g} / \mathrm{ml}$, corresponding to $400 \mathrm{nM}$ ) into $150 \mu \mathrm{L}$ of DMEM medium (without SVF) combined with polyethyleneimine (PEI, reference 408727, average MW: 25000, Sigma-Aldrich), with an hpdODN/PEI ratio of 1:1. For immunocytochemistry, liposomes prepared as previously described [23] were used (at $2 \mu \mathrm{g}$ of cationic lipid). After $6 \mathrm{~h}$ at $37^{\circ} \mathrm{C}$ in a humidified $5 \% \mathrm{CO}_{2}$ incubator, the cells were placed in fresh serum-containing medium. Cells were examined after $48 \mathrm{~h}$ in the humidified incubator.

\section{Flow cytometry and cell viability}

To measure cell death, cells were resuspended in annexin $\mathrm{V}$-binding buffer, incubated with $5 \mu \mathrm{L}$ of propidium iodide (PI, BD Pharmingen, Morangis, France) and subjected to flow cytometry analysis, using a FACS 
Canto II Flow Cytometer (BD). To enable selective analysis of the cells that had incorporated the various hpdODNs, fluorescein-labelled hpdODNs were used. Fluorescein-labelled cells were analyzed for PI incorporation (red channel) or annexin V labelling (APC, red channel). A cell death index was established through computation of averages.

\section{Gel electrophoresis, western blotting}

Cells were washed in Phosphate-Buffered Saline (PBS), lysed in sodium dodecyl sulfate (SDS) sample buffer (50 mM Tris-HCl pH 6.8 (Bio-Rad, Marnes-la-Coquette, France), 2\% SDS (Sigma-Aldrich), 20\% glycerol (Prolabo, Fontenay-sous-Bois, France), $1 \mathrm{mM}$ sodium vanadate $\left(\mathrm{Na}_{3} \mathrm{VO}_{4}\right.$, Labosi, Elancourt, France), $1 \mathrm{mM}$ dithiothreitritol (DTT) (Merck, Fontenay-sous-Bois, France) and 0.01\% bromophenol blue (Sigma-Aldrich)), sonicated and stored at $-70^{\circ} \mathrm{C}$. Proteins $(50 \mu \mathrm{g})$ were separated on SDS polyacrylamide gels (PAGE) $(10 \%)$ and transferred onto nitrocellulose membranes; membranes blocked with dry skimmed milk (5\%) in Tris Buffered Saline (TBS) were incubated with antibody overnight at $4^{\circ} \mathrm{C}$. Anti-phospho-STAT1, anti-STAT1 and anti-STAT3 (1:1000) (Cell Signaling, Ozyme, Montigny-le-Bretonneux, France), anti-cyclin D1 and anti-IRF1 (1:1000) (Santa Cruz, Tebu-bio, Le Perray en Yvelines, France) were used. Blots were washed in TBS with Tween (0.05\%) (TBS-T), incubated with peroxidase-coupled goat anti-mouse (Santa Cruz) or goat anti-rabbit (Upstate, Ozyme) secondary antibody $(1: 20,000)$, washed in TBS-T and revealed by chemiluminescence (LumiGLO reagent and peroxide; Cell Signaling) and autoradiography (X-Omat R film; Kodak). When necessary, membranes were stripped with Blot Restore Kit (Chemicon International, Millipore, Saint-Quentin-en-Yvelines, France) and reprobed with anti-tubulin (Cell Signaling) or anti-actin (Santa Cruz) antibody to ensure equal loading of the gels. Prestained molecular weight standards (Fermentas, Saint-Rémy-lès-Chevreuse, France) were used.

\section{Oligodeoxynucleotide pull-down}

For in-cell hpdODN pull-down assays, cells were transfected with the biotinylated hpdODNs $(1 \mu \mathrm{g} / \mathrm{ml}, 6 \mathrm{~h})$, as described under oligonucleotide transfection, and then lysed in cell lysis buffer (1\% NP40, $50 \mathrm{mM}$ Hepes, $\mathrm{pH}$ 7.6, $140 \mathrm{mM} \mathrm{NaCl}$ ) containing salmon sperm DNA (1 $\mu \mathrm{g}$ /assay). Protein concentration was measured in the samples. Extracts $(250 \mu \mathrm{g}$ of protein per tube) were recovered on avidin-sepharose beads $(50 \mu \mathrm{L})$ (NeutrAvidin, Pierce); beads were incubated for $30 \mathrm{~min}$ at $4^{\circ} \mathrm{C}$ in binding buffer (1\% NP40, $50 \mathrm{mM}$ Hepes, pH 7.6, 140 $\mathrm{mM} \mathrm{NaCl}$ ). After washing with binding buffer, complexes were eluted in SDS sample buffer, separated on SDS-PAGE (8\%), and subjected to immunoblotting using anti-STAT1 or anti-STAT3 antibodies (Cell Signaling) and processed as above.

\section{Immunocytochemistry}

Cells were grown at 50-60\% confluence in 8-well plates (Lab-Tek, Nunc, Rochester, USA) to a density of $10^{5}$ cells/ $\mathrm{ml}$. Cells were transfected with fluorescein-labelled hpdODNs, incubated (6 to $16 \mathrm{~h}$ ), washed in PBS, fixed with $3.7 \%$ formaldehyde for $15 \mathrm{~min}$, permeabilized in $0.1 \%$ Triton X-100 for 15 min and incubated in 5\% FCS-0.1\% Tween- PBS for $1 \mathrm{~h}$. Cells were stained with anti-STAT3 or anti-STAT1 antibody (Cell Signaling) (1:100) for $2 \mathrm{~h}$, then stained with an Alexa fluor 546-labeled secondary antibody (Invitrogen) (1:200) for $90 \mathrm{~min}$. Cells, counterstained with 4', 6'-diamidino-2-phenylindole (DAPI), were mounted onto glass slides with Vectashield (Vectorlabs, Clinsciences, Montrouge, France). Fluorescence images were acquired using a Zeiss Axioplan 2 Deconvolution microscope (Carl Zeiss, Le Pecq, France) and analyzed with Metafer4 (Metasystems, Altlussheim, Germany).

\section{Additional material}

Additional file 1: Table S1 List of the contacts between STAT1, STAT3 and DNA. Contacts were evaluated using the "Find clashes/ contacts" routine in Chimera with the default parameters (cut off $=-0.4$ $\AA$ and allowance for potentially hydrogen-bonded pairs $=0.0 \AA$ ). These values allow the van der Waals radii in atom pairs to be taken into account rather than interatomic distances alone.

\section{Acknowledgements}

We thank Martin Zacharias (Technical University Munich, Germany) for critical advice and fruitful discussions at the onset of this study. IS was supported by a one-year ( $3^{\text {rd }} \mathrm{PhD}$ year) grant from the Association pour la Recherche sur le Cancer (ARC). This work was funded in part by grants from OSEO (France), the Ligue nationale contre le cancer (France) and the Ligue départementale contre le cancer (comité du Val d'Oise).

\section{Author details}

${ }^{1}$ INSERM, Unité 978, Bobigny 93017, France. '2Université Paris 13, UFR SMBH, Bobigny 93017, France. ${ }^{3}$ AP-HP, Hôpital Avicenne, Service de biochimie, Bobigny 93009, France. ${ }^{4}$ AP-HP, Hôpital Avicenne, Service d'hématologie biologique, Bobigny 93009, France. ${ }^{5}$ Moscow State University, Moscow, Russia. ${ }^{6}$ CNRS UMR 7244, CSPBAT, Bobigny 93017, France. ${ }^{7}$ Université Paris 6, CNRS FRE 3207 ANBio $\phi$, Paris 75005, France.

\section{Authors' contributions}

IS, PL, VM, SLC, JAHC and RF made substantial contributions to conception, design, and data acquisition, analysis and interpretation; NVB and FBM were involved in revising the manuscript for important intellectual content; and IS, PL, VM, JAHC, SLC, NVB, FBM and RF gave final approval of the version to be published. All authors read and approved the final manuscript.

\section{Competing interests}

The authors declare that they have no competing interests.

Received: 23 December 2011 Accepted: 16 March 2012 Published: 16 March 2012 


\section{References}

1. Schindler C, Levy DE, Decker T: JAK-STAT signaling: from interferons to cytokines. J Biol Chem 2007, 282(28):20059-20063.

2. Qing Y, Stark GR: Alternative activation of STAT1 and STAT3 in response to interferon-gamma. J Biol Chem 2004, 279(40):41679-41685.

3. Santos Cl, Costa-Pereira AP: Signal transducers and activators of transcription-from cytokine signalling to cancer biology. Biochim Biophys Acta 2011, 1816(1):38-49

4. Frank DA: STAT3 as a central mediator of neoplastic cellular transformation. Cancer Lett 2007, 251(2):199-210.

5. Jarnicki A, Putoczki T, Ernst M: Stat3: linking inflammation to epithelial cancer-more than a "gut" feeling? Cell Div 2010, 5:14.

6. Bromberg JF: Activation of STAT proteins and growth control. Bioessays 2001, 23(2):161-169.

7. Bromberg J: Stat proteins and oncogenesis. J Clin Invest 2002, 109(9):1139-1142.

8. Al Zaid Siddiquee K, Turkson J: STAT3 as a target for inducing apoptosis in solid and hematological tumors. Cell Res 2008, 18(2):254-267.

9. de la Iglesia N, Konopka G, Puram SV, Chan JA, Bachoo RM, You MJ, Levy DE, Depinho RA, Bonni A: Identification of a PTEN-regulated STAT3 brain tumor suppressor pathway. Genes Dev 2008, 22(4):449-462.

10. de la Iglesia N, Konopka G, Lim KL, Nutt CL, Bromberg JF, Frank DA Mischel PS, Louis DN, Bonni A: Deregulation of a STAT3-interleukin 8 signaling pathway promotes human glioblastoma cell proliferation and invasiveness. J Neurosci 2008, 28(23):5870-5878.

11. Stephanou A, Latchman DS: STAT-1: a novel regulator of apoptosis. Int $J$ Exp Pathol 2003, 84(6):239-244.

12. Najjar I, Fagard R: STAT1 and pathogens, not a friendly relationship. Biochimie 2010, 92:425-444.

13. Lin J, Buettner R, Yuan YC, Yip R, Horne D, Jove R, Vaidehi N: Molecular dynamics simulations of the conformational changes in signal transducers and activators of transcription, Stat1 and Stat3. J Mol Graph Model 2009, 28(4):347-356.

14. Kramer $\mathrm{OH}$, Heinzel $\mathrm{T}$ : Phosphorylation-acetylation switch in the regulation of STAT1 signaling. Mol Cell Endocrinol 2010, 315(1-2):40-48.

15. Kovacic B, Stoiber D, Moriggl R, Weisz E, Ott RG, Kreibich R, Levy DE, Beug $H$, Freissmuth M, SexI V: STAT1 acts as a tumor promoter for leukemia development. Cancer Cell 2006, 10(1):77-87.

16. Page BD, Ball DP, Gunning PT: Signal transducer and activator of transcription 3 inhibitors: a patent review. Expert Opin Ther Pat 2011, 27(6):e293-300.

17. Aggarwal BB, Kunnumakkara AB, Harikumar KB, Gupta SR, Tharakan ST, Koca C, Dey S, Sung B: Signal transducer and activator of transcription-3, inflammation, and cancer: how intimate is the relationship? Ann N Y Acad Sci 2009, 1171:59-76.

18. Yue P, Turkson J: Targeting STAT3 in cancer: how successful are we? Expert Opin Investig Drugs 2009, 18(1):45-56.

19. Schust J, Sperl B, Hollis A, Mayer TU, Berg T: Stattic: a small-molecule inhibitor of STAT3 activation and dimerization. Chem Biol 2006, 13(11):1235-1242.

20. Miyoshi K, Takaishi M, Nakajima K, Ikeda M, Kanda T, Tarutani M, liyama T, Asao N, DiGiovanni J, Sano S: Stat3 as a therapeutic target for the treatment of psoriasis: a clinical feasibility study with STA-21, a Stat3 inhibitor. J Invest Dermatol 2011, 131(1):108-117.

21. Leong PL, Andrews GA, Johnson DE, Dyer KF, Xi S, Mai JC, Robbins PD, Gadiparthi S, Burke NA, Watkins SF, et al: Targeted inhibition of Stat3 with a decoy oligonucleotide abrogates head and neck cancer cell growth. Proc Natl Acad Sci USA 2003, 100(7):4138-4143.

22. Tadlaoui Hbibi A, Laguillier C, Souissi I, Lesage D, Le Coquil S, Cao A, Metelev V, Baran-Marszak F, Fagard R: Efficient killing of SW480 colon carcinoma cells by a STAT3 hairpin decoy oligodeoxynucleotide. Interference with interferon gamma-STAT1-mediated killing. FEBS Journal 2009, 276:2505-2515.

23. Souissi I, Najjar I, Ah-Koon L, Schischmanoff PO, Lesage D, Le Coquil S, Roger C, Dusanter-Fourt I, Varin-Blank N, Cao A, et al: A STAT3-decoy oligonucleotide induces cell death in a human colorectal carcinoma cell line by blocking nuclear transfer of STAT3 and STAT3-bound NF-kappaB. BMC Cell Biol 2011, 12:14

24. Kumar A, Commane M, Flickinger TW, Horvath CM, Stark GR: Defective TNF-alpha-induced apoptosis in STAT1-null cells due to low constitutive levels of caspases. Science 1997, 278(5343):1630-1632.
25. Thomas M, Finnegan CE, Rogers KM, Purcell JW, Trimble A, Johnston PG Boland MP: STAT1: a modulator of chemotherapy-induced apoptosis. Cancer Res 2004, 64(22):8357-8364.

26. Schindler C: STATs as activators of apoptosis. Trends Cell Biol 1998, 8(3):97-98.

27. Sironi JJ, Ouchi T: STAT1-induced apoptosis is mediated by caspases 2, 3, and 7. J Biol Chem 2004, 279(6):4066-4074.

28. Townsend PA, Scarabelli TM, Davidson SM, Knight RA, Latchman DS, Stephanou A: STAT-1 interacts with p53 to enhance DNA damageinduced apoptosis. J Biol Chem 2004, 279(7):5811-5820.

29. Baran-Marszak F, Feuillard J, Najjar I, Le Clorennec C, Bechet JM, DusanterFourt I, Bornkamm GW, Raphael M, Fagard R: Differential roles of STAT1alpha and STAT1beta in fludarabine-induced cell cycle arrest and apoptosis in human B cells. Blood 2004, 104(8):2475-2483.

30. Wall L, Burke F, Barton C, Smyth J, Balkwill F: IFN-gamma induces apoptosis in ovarian cancer cells in vivo and in vitro. Clin Cancer Res 2003, 9(7):2487-2496.

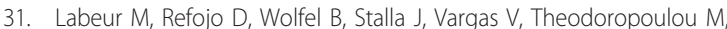
Buchfelder M, Paez-Pereda M, Arzt E, Stalla GK: Interferon-gamma inhibits cellular proliferation and ACTH production in corticotroph tumor cells through a novel janus kinases-signal transducer and activator of transcription 1/nuclear factor-kappa B inhibitory signaling pathway. $J$ Endocrinol 2008, 199(2):177-189.

32. Ning Y, Riggins RB, Mulla JE, Chung H, Zwart A, Clarke R: IFNgamma restores breast cancer sensitivity to fulvestrant by regulating STAT1, IFN regulatory factor $1, \mathrm{NF}-\mathrm{kappaB}, \mathrm{BCL} 2$ family members, and signaling to caspase-dependent apoptosis. Mol Cancer Ther 2010, 9(5):1274-1285.

33. Darnell JE Jr, Kerr IM, Stark GR: Jak-STAT pathways and transcriptional activation in response to IFNs and other extracellular signaling proteins. Science 1994, 264(5164):1415-1421.

34. Wagner BJ, Hayes TE, Hoban CJ, Cochran BH: The SIF binding element confers sis/PDGF inducibility onto the c-fos promoter. EMBO $J 1990$ 9(13):4477-4484

35. de Koning JP, Dong F, Smith L, Schelen AM, Barge RM, van der Plas DC, Hoefsloot LH, Lowenberg B, Touw IP: The membrane-distal cytoplasmic region of human granulocyte colony-stimulating factor receptor is required for STAT3 but not STAT1 homodimer formation. Blood 1996, 87(4):1335-1342

36. Horvath CM, Wen Z, Darnell JE Jr: A STAT protein domain that determines DNA sequence recognition suggests a novel DNA-binding domain. Genes Dev 1995, 9(8):984-994.

37. Seidel HM, Milocco LH, Lamb P, Darnell JE Jr, Stein RB, Rosen J: Spacing of palindromic half sites as a determinant of selective STAT (signal transducers and activators of transcription) DNA binding and transcriptional activity. Proc Natl Acad Sci USA 1995, 92(7):3041-3045.

38. Lamb P, Seidel HM, Haslam J, Milocco L, Kessler LV, Stein RB, Rosen J: STAT protein complexes activated by interferon-gamma and gp130 signaling molecules differ in their sequence preferences and transcriptional induction properties. Nucleic Acids Res 1995, 23(16):3283-3289.

39. Ehret GB, Reichenbach $P$, Schindler U, Horvath CM, Fritz S, Nabholz M Bucher P: DNA binding specificity of different STAT proteins. Comparison of in vitro specificity with natural target sites. J Biol Chem 2001, 276(9):6675-6688.

40. Leung $T H$, Hoffmann A, Baltimore D: One nucleotide in a kappaB site can determine cofactor specificity for NF-kappaB dimers. Cell 2004, 118(4):453-464.

41. Nelson HC, Finch JT, Luisi BF, Klug A: The structure of an oligo(dA).oligo (dT) tract and its biological implications. Nature 1987, 330(6145):221-226.

42. Rohs R, West SM, Sosinsky A, Liu P, Mann RS, Honig B: The role of DNA shape in protein-DNA recognition. Nature 2009, 461(7268):1248-1253.

43. Rohs $R$, Jin X, West SM, Joshi R, Honig B, Mann RS: Origins of specificity in protein-DNA recognition. Annu Rev Biochem 2010, 79:233-269.

44. Chen X, Vinkemeier U, Zhao Y, Jeruzalmi D, Darnell JE Jr, Kuriyan J: Crystal structure of a tyrosine phosphorylated STAT-1 dimer bound to DNA. Cell 1998, 93(5):827-839.

45. Becker S, Groner B, Muller CW: Three-dimensional structure of the Stat3beta homodimer bound to DNA. Nature 1998, 394(6689):145-151.

46. Pettersen EF, Goddard TD, Huang CC, Couch GS, Greenblatt DM, Meng EC, Ferrin TE: UCSF Chimera-a visualization system for exploratory research and analysis. J Comput Chem 2004, 25(13):1605-1612. 
47. Rouyez MC, Lestingi M, Charon M, Fichelson S, Buzyn A, Dusanter-Fourt I: IFN regulatory factor- 2 cooperates with STAT1 to regulate transporter associated with antigen processing-1 promoter activity. J Immuno/ 2005 174(7):3948-3958.

48. Chin YE, Kitagawa M, Kuida K, Flavell RA, Fu XY: Activation of the STAT signaling pathway can cause expression of caspase 1 and apoptosis. Mol Cell Biol 1997, 17(9):5328-5337.

49. Epling-Burnette PK, Zhong B, Bai F, Jiang K, Bailey RD, Garcia R, Jove R, Djeu JY, Loughran TP Jr, Wei S: Cooperative regulation of Mcl-1 by Janus kinase/stat and phosphatidylinositol 3-kinase contribute to granulocytemacrophage colony-stimulating factor-delayed apoptosis in human neutrophils. J Immunol 2001, 166(12):7486-7495.

50. Geller J, Petak I, Szucs KS, Nagy K, Tillman DM, Houghton JA: Interferongamma-induced sensitization of colon carcinomas to ZD9331 targets caspases, downstream of Fas, independent of mitochondrial signaling and the inhibitor of apoptosis survivin. Clin Cancer Res 2003, 9(17):6504-6515.

51. Geller Jl, Szekely-Szucs K, Petak I, Doyle B, Houghton JA: P21Cip1 is a critical mediator of the cytotoxic action of thymidylate synthase inhibitors in colorectal carcinoma cells. Cancer Res 2004, 64(17):6296-6303.

52. Hizver J, Rozenberg H, Frolow F, Rabinovich D, Shakked Z: DNA bending by an adenine-thymine tract and its role in gene regulation. Proc Natl Acad Sci USA 2001, 98(15):8490-8495.

53. Costa-Pereira AP, Tininini S, Strobl B, Alonzi T, Schlaak JF, Is'harc $H$, Gesualdo I, Newman SJ, Kerr IM, Poli V: Mutational switch of an IL-6 response to an interferon-gamma-like response. Proc Natl Acad Sci USA 2002, 99(12):8043-8047.

54. Tanabe Y, Nishibori T, Su L, Arduini RM, Baker DP, David M: Cutting edge: role of STAT1, STAT3, and STAT5 in IFN-alpha beta responses in T lymphocytes. J Immunol 2005, 174(2):609-613.

55. Gimeno R, Lee CK, Schindler C, Levy DE: Stat1 and Stat2 but not Stat3 arbitrate contradictory growth signals elicited by alpha/beta interferon in T lymphocytes. Mol Cell Biol 2005, 25(13):5456-5465.

56. Regis G, Pensa S, Boselli D, Novelli F, Poli V: Ups and downs: The STAT1: STAT3 seesaw of Interferon and gp130 receptor signalling. Semin Cell Dev Biol 2008, 19(4):351-359.

57. Schiavone D, Avalle L, Dewilde S, Poli V: The immediate early genes Fos and Egr1 become STAT1 transcriptional targets in the absence of STAT3. FEBS Lett 2011, 585(15):2455-2460

58. Adach-Kilon A, Swiatek-Machado K, Kaminska B, Dabrowski M: Signal transducer and activator of transcription 1 (Stat1) maintains basal mRNA expression of pro-survival stat3-target genes in glioma C6 cells. J Cell Biochem 2011, 112(12):3685-3694.

59. Vinkemeier U, Cohen SL, Moarefi I, Chait BT, Kuriyan J, Darnell JE Jr: DNA binding of in vitro activated Stat1 alpha, Stat1 beta and truncated Stat1: interaction between $\mathrm{NH}$ 2-terminal domains stabilizes binding of two dimers to tandem DNA sites. Embo J 1996, 15(20):5616-5626.

60. Yoshizawa S, Ueda T, Ishido Y, Miura K, Watanabe K, Hirao I: Nuclease resistance of an extraordinarily thermostable mini-hairpin DNA fragment, $d(G C G A A G C)$ and its application to in vitro protein synthesis. Nucleic Acids Res 1994, 22(12):2217-2221.

61. Hirao I, Kawai G, Yoshizawa S, Nishimura Y, Ishido Y, Watanabe K, Miura K: Most compact hairpin-turn structure exerted by a short DNA fragment, $\mathrm{d}(\mathrm{GCGAAGC})$ in solution: an extraordinarily stable structure resistant to nucleases and heat. Nucleic Acids Res 1994, 22(4):576-582.

62. Santini GP, Cognet JA, Xu D, Singarapu KK, Herve du Penhoat C: Nucleic acid folding determined by mesoscale modeling and NMR spectroscopy: solution structure of d(GCGAAAGC). J Phys Chem B 2009, 113(19):6881-6893.

doi:10.1186/1476-4598-11-12

Cite this article as: Souissi et al:: A STAT3-inhibitory hairpin decoy oligodeoxynucleotide discriminates between STAT1 and STAT3 and induces death in a human colon carcinoma cell line. Molecular Cancer 2012 11:12.

\section{Submit your next manuscript to BioMed Central and take full advantage of:}

- Convenient online submission

- Thorough peer review

- No space constraints or color figure charges

- Immediate publication on acceptance

- Inclusion in PubMed, CAS, Scopus and Google Scholar

- Research which is freely available for redistribution

Submit your manuscript at www.biomedcentral.com/submit
Biomed Central 